\title{
Aircraft Spareparts Demand Forecasting based on Multiple ARIMA Model
}

\author{
Houju Xin ${ }^{1, a}$, Yang Cui ${ }^{1,}$, Xinbin Liu ${ }^{1,}$, Shujian Luo ${ }^{2, \text { a }}$
}

${ }^{1}$ Department of Air Materials Management, Air Force Logistics College, Xuzhou 221000, China

${ }^{2}$ Troop 95106, Zhuzhou 412000, China

aemail:1165193244@qq.com

Keywords: Time Series, Demand forecasting, Parameter estimation, White noise sequence.

\begin{abstract}
In order to predict the uncertain demand for aircraft spare parts, a multiple ARIMA model is used to solve this problem by time series forecasting system in SPSS. The prediction result and its applications are discussed. This method is simple, practical and convenient for spreading.
\end{abstract}

\section{Introduction}

With the air force's facelift and aircraft upgrading, more and more types of aviation equipment, valued at more expensive, how equipment based on historical consumption data to accurately forecast future equipment needs, which not only improves the Air Materiel Support the degree of precision, reduce inventory, to avoid the waste of time-sensitive due to equipment produced, and increased air material guarantee predictability, lay the foundation for a variety of missions to complete. The demand for certain types of aircraft materials, can over time, forming a sequence, we called time-series aircraft materials demand. For certain types of aircraft materials, the demand for a certain period of time, is uncertain, it is influenced by the intensity of military training, environment and climate, seasonal and other factors. Therefore, the time series may, over time, show a certain trend, which also may be affected by seasonal factors, showed some seasonal, such as the rainy season training intensity reduction of the consumption of the equipment is small, then the corresponding reduction in demand. The current demand for air material forecasting, they use a regression method, the moving average method, and the processing and forecasting methods, the lack of seasonal considerations, and the use of time-series models $\operatorname{ARIMA}(p, d, q)(P, D, Q)^{s}$ variety of materials can affect the demand for aircraft factors to consider, for the short-term forecast is better.

\section{$\operatorname{ARIMA}(p, d, q)(P, D, Q)^{s}$ Model}

If $\left\{y_{t}\right\}$ time series is a stationary, you can use auto-regressive moving average model $A R M A(p, q)$ realization of modeling and forecasting, however, if the non-stationary time series trend of time series, $\operatorname{ARMA}(p, q)$ model cannot be directly established after smoothing can handle them. Here smoothing treatment is generally treated with a difference, after the model in mind for differential treatment $\operatorname{ARIMA}(p, d, q)$, difference is the order of $d, B^{k}$ note for the $k$ order lag operator, $B^{k} y_{t}=y_{t-k}$ if $k=1$, then $B y_{t}=y_{t-1} \cdot(1-B)^{d}$ Form is represented by the difference, if $d=1,(1-B) y_{t}=y_{t}-y_{t-1}$ is the first difference. Values and the seasons change some sequences related to and often also for excluding the impact of seasonal, but also for the season so the difference can be expressed as $\left(1-B^{s}\right)^{D}$, if $D=1$, then $\left(1-B^{s}\right) y_{t}=y_{t}-y_{t-s}$ is a first-order differential season, if the monthly seasonal differencing $s=12$, If the quarterly seasonal differencing $s=4$. In order to consider all the circumstances, to consider the form of the following models:

$$
\phi(B) U(B)(1-B)^{d}\left(1-B^{s}\right)^{D} y_{t}=\theta(B) V(B) \varepsilon_{t}
$$

This model is the $\operatorname{ARIMA}(p, d, q)(P, D, Q)^{s}$ model, autoregressive moving average model of promotion. Then $\phi(B)=1-\phi_{1} B-\phi_{2} B^{2}-\cdots-\phi_{p} B^{p}$, is $\mathrm{p}$ autoregressive operator, 
$\theta(B)=1-\theta_{1} B-\theta_{2} B^{2}-\cdots-\theta_{p} B^{q}$, is $\mathrm{q}$ autoregressive operator, $(1-B)^{d}$ is d-order differential operator, $U(B)=1-u_{1} B^{s}-u_{2} B^{2 s}-\cdots-u_{P} B^{P s}$ season is a P-order autoregressive mobile operator, $V(B)=1-v_{1} B^{s}-v_{2} B^{2 s}-\cdots-v_{Q} B^{Q s}$ season is a Q-order moving average operator, $\left(1-B^{s}\right)^{D}$ season is

a D-order differential operator, then $\phi_{1}, \phi_{2}, \cdots, \phi_{p}, \theta_{1}, \theta_{2}, \cdots, \theta_{q}, u_{1}, u_{2}, \cdots, u_{P}, v_{1}, v_{2}, \cdots, v_{Q}$ are parameters to be estimated.

\section{Step $\operatorname{ARIMA}(p, d, q)(P, D, Q)^{s}$ model predictions}

The first step: transformed into a smooth sequence. Strict judging stability more difficult sequences, can make use of the image, if the image no trend, no periodicity can be broadly considered stationary series, can also use the autocorrelation function ACF, if the autocorrelation function ACF increased with time lag, and quickly goes to zero, it is considered that the series is stationary. Non-stationary sequence, if you have a strong trend, you can gradually phase difference, the difference in the number of by-period decision model values, if the sequence is more obvious cyclical, seasonal difference can be achieved through stability, seasonal differencing the order is in the model D. Step Two: Determine the form of the model. Primarily order to identify practical ways is through correlation function and partial correlation functions and their graphs, find the appropriate $\mathrm{p}$ and $\mathrm{q}$. The third step: to estimate the parameters of the model. And selected according to the value of the estimated model parameters contained autoregressive and moving average terms. Since the method and procedure for solving stochastic time series model is generally more complex, generally need to use statistical software, the paper on the use of specialized statistical software SPSS. Step Four: Model diagnostics. After the selected model and estimate its parameters, then we must look at the effect of the model is a good fit. A simple test is to determine the estimated residuals from this model are for white noise: If it is, you can accept the fitted model; if not, go back to re-fit. Step Five: model predictions. In the fitted model suitable base can be predicted using the model, the actual calculation is more complex, is achieved by means of the use of statistical software.

\section{Empirical Analysis}

Some aviation materials January 2000 to December 2004 the demand in Table 1.

Table 1: A flight equipment demand in January 2000 to December 2004

\begin{tabular}{|c|c|c|c|c|c|c|c|c|c|c|c|c|}
\hline Month & 2001.1 & 2 & 3 & 4 & 5 & 6 & 7 & 8 & 9 & 10 & 11 & 12 \\
\hline Demand Y & 15 & 54 & 85 & 93 & 51 & 22 & 9 & 9 & 11 & 75 & 54 & 22 \\
\hline Month & 2002.1 & 2 & 3 & 4 & 5 & 6 & 7 & 8 & 9 & 10 & 11 & 12 \\
\hline Demand Y & 22 & 60 & 88 & 95 & 56 & 23 & 9 & 10 & 14 & 81 & 56 & 23 \\
\hline Month & 2003.1 & 2 & 3 & 4 & 5 & 6 & 7 & 8 & 9 & 10 & 11 & 12 \\
\hline Demand Y & 23 & 64 & 90 & 99 & 60 & 30 & 11 & 12 & 15 & 85 & 59 & 25 \\
\hline Month & 2004.1 & 2 & 3 & 4 & 5 & 6 & 7 & 8 & 9 & 10 & 11 & 12 \\
\hline Demand Y & 25 & 70 & 93 & 98 & 62 & 32 & 13 & 14 & 19 & 90 & 61 & 28 \\
\hline
\end{tabular}

Air Materiel Y Draw a timing diagram of the demand, there is a clear trend in the time series nature, be a difference, there are significant seasonal, then it's a seasonal difference, the last from the first-order differential and again serialize the season after a first-order differential timing diagram to see, in addition to the data in October 2001 appeared abnormal, other data base has stabilized, the new sequence generation can be seen as a smooth sequence. We used SPSS software to calculate the first-order differential and difference sequence season after a first-order autocorrelation function and 
the partial autocorrelation function, the results from the software and PAC ACF diagram can be seen, the sample autocorrelation function and the partial autocorrelation function, very quickly fall within random intervals, so the time series trend basically eliminated, but the autocorrelation function and the partial autocorrelation function values associated tailing is still large. To compensate for this, we had to deal with time-series then seasonal autoregressive and moving average, in order to reflect the simplicity of the model, therefore, in model $\operatorname{ARIMA}(p, d, q)(P, D, Q)^{s}$, select $P=1, Q=1$.In Model $\operatorname{ARIMA}(p, d, q)(P, D, Q)^{s}$, as a result of the first-order differential, $d=1$ has conducted a first-order differential season, take $D=1$. Will use the following model:

$$
\phi(B)\left(1-u_{1} B^{12}\right)\left(1-B^{12}\right)(1-B) y_{t}=\theta(B)\left(1-v_{1} B^{12}\right) \varepsilon_{t}
$$

For the first time by a sequence of differential and difference after the first season, and calculate the mean of -0.04 standard deviation of 3.476. After a sequence difference autocorrelation function and partial autocorrelation function, there were tailing, according to their value, think $p=2, q=1$ more appropriate, we use SPSS, take $d=1, P=1, D=1, Q=1$, wish for $(p, q)$ few more choices, the results in Table 2.

Table 2: (p, q) comparing the test results for different values

\begin{tabular}{|c|c|c|c|c|}
\hline$(\mathrm{p}, \mathrm{q})$ & Standard error & Log likelihood & AIC & SBC \\
\hline$(1,1)$ & 2.5375693 & -110.84676 & 229.69352 & 237.09411 \\
\hline$(2,1)$ & 2.4858136 & -109.70991 & 229.41982 & 238.67056 \\
\hline$(2,2)$ & 2.5009555 & -109.58746 & 231.17491 & 242.2758 \\
\hline$(3,1)$ & 2.7722286 & -112.9447 & 237.8894 & 248.99028 \\
\hline$(3,2)$ & 2.5243877 & -109.63275 & 233.26549 & 246.21653 \\
\hline$(3,3)$ & 2.4892184 & -108.31278 & 232.62557 & 247.42675 \\
\hline
\end{tabular}

As can be seen from Table 2, $(p, q)$ take(2,1), Standard error least, AIC least, SBC the second smallest, Log likelihood the best, though not the biggest, but other values and the number of take-likelihood estimate when the value of the gap is very small, a comprehensive comparison, $(p, q)$ get $(2,1)$ when. $(p, q)$ Parameter estimation takes $(2,1)$ when the results in the table below:

\begin{tabular}{|c|c|c|c|c|c|}
\hline$(\mathrm{p}, \mathrm{q})$ & $\phi_{1}$ & $\phi_{2}$ & $\theta_{1}$ & $u_{1}$ & $v_{1}$ \\
\hline$(2,1)$ & 0.13682884 & -0.11361482 & 0.99433274 & 0.84767265 & 0.63106341 \\
\hline
\end{tabular}

Then the model is:

$$
\begin{aligned}
& \left(1-0.13682884 \mathrm{~B}+0.11361482 \mathrm{~B}^{2}\right) \cdot\left(1-0.84767265 B^{12}\right) \cdot\left(1-B^{12}\right) \cdot(1-B) y_{t} \\
& =(1-0.99433274 B) \cdot\left(1-0.63106341 B^{12}\right) \varepsilon_{t}
\end{aligned}
$$

Tested for residual autocorrelation function and partial autocorrelation functions fall into the random interval (see Figure 3), the residual white noise sequence, and indicating better model predictions. -0.3804061 Residuals mean, standard deviation of 2.56924639.

Finally forecasting January 2005 to December of values in Table 3.

Table 3: January 2005 - December predictive value

\begin{tabular}{|c|c|c|c|c|c|c|}
\hline Month & 1 & 2 & 3 & 4 & 5 & 6 \\
\hline Predictive value & 28.24707 & 74.16390 & 96.00452 & 100.19483 & 65.01170 & 35.12358 \\
\hline Month & 7 & 8 & 9 & 10 & 11 & 12 \\
\hline Predictive value & 15.23597 & 16.26256 & 21.82723 & 94.76048 & 63.78052 & 30.63575 \\
\hline
\end{tabular}


We put the original sequence (demand Y), the analog value, the predicted value and the residual series painted in the same graph (Figure 1), it can be seen, the original sequence and simulation sequences fit better.

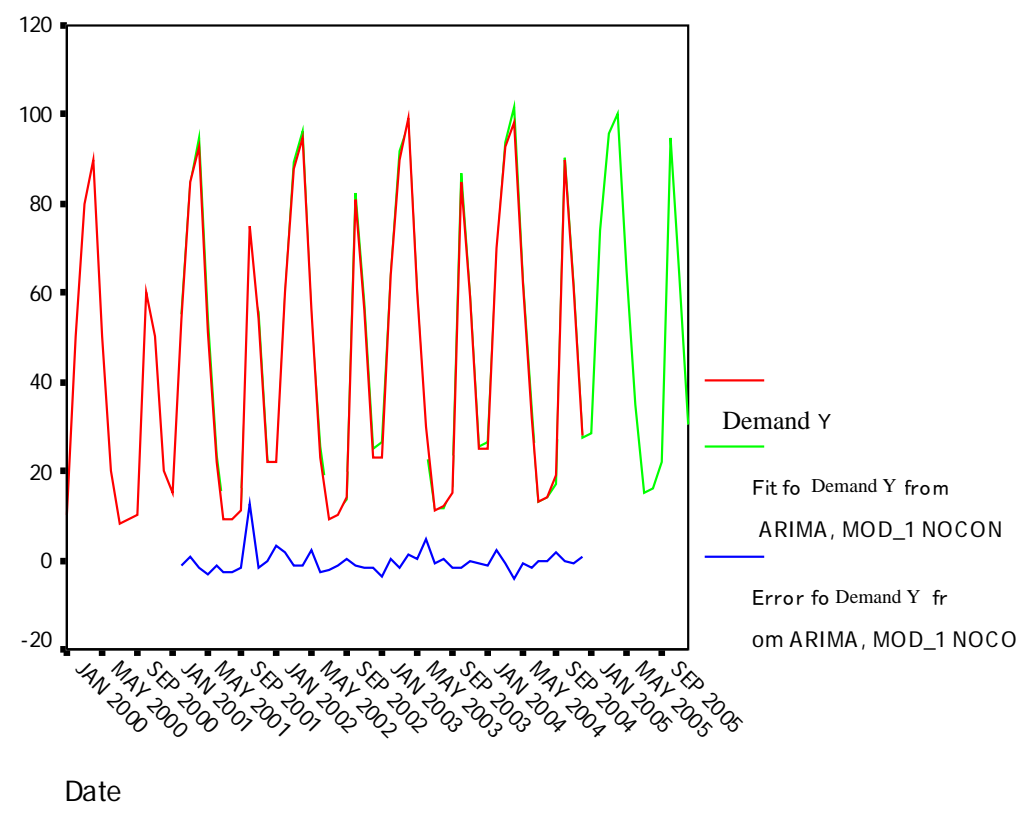

Figure 1. The original sequence, analog values, predicted values and residuals sequence comparison chart

\section{Summary}

Air Materiel paper use actual demand data, seasonally-by-stage differential and difference, converted into stationary time series, fully consider the impact of the seasonal demand for aircraft materials, considering various factors Air Materiel demand by Extensional autoregressive moving average model - multiplicative seasonal model a, and then use statistical software, the future of aviation material needs to do short-term prediction, the prediction error is small, high precision. The method uses statistical software; computing and testing are complete with a machine that is convenient and practical.

\section{References}

[1] Guoxiang. Statistical forecasting and decision-making [M] Shanghai: Shanghai University of Finance and Economics Press, 2010.

[2] Tang Wei. Air material demand forecasting equipment maintenance and support of research [D] Wuhan: Wuhan University of Technology, 2011.

[3] Research [J] Wang, Wang Zibin, Li Li, etc. Air Materiel repair funds configuration. Qingdao University (Natural Science), 2010, 23 (3), p. 52-55.

[4] Gu Zhiyu, Kang Rui prediction method is based on the consumption of spare parts ARIMA model [J]. Ordnance Industry Automation, 2010, 28 (6), p. 29-31. 